gerechnet, die entsprechenden Messungen entnommen wurden. Der Verlauf der Luftspaltinduktion und der Stegstreufluß wurde durch gleichstromerregte Nachbildung der beiden Grenzstellungen des Drehfeldes ermittelt. Dadurch wurde die Unsicherheit bei der Annahme der Magnetisierungskurven für die durch das Stanzen magnetisch verhärteten schmalen Streustege vermieden. Der Käfigläufer wurde mit Kupferstäben und hartverlöteten Endringen aufgebaut, um den Einfluß der bei aluminiumvergossenen Kurzschlußläufern mangelhaften Stabisolierung gegen das Eisen zu eliminieren. Eine vollkommene Vorausberechnung eines Motors mit ausgeprägten Polen und geschlossenen Streustegen wird insbesondere bei Verwendung eines aluminiumvergossenen Kurzschlußläufers nicht mit einer derartigen Genauigkeit wie bei dem angeführten Beispiel möglich sein. Hier sollte jedoch die Aufgabe der Nachrechnung sein, die Richtigkeit der theoretischen Voraussagen zu bestätigen. Ferner erkennt man den noch recht großen Einfluß der schädlichen 3. Feldoberwelle in der DrehmomentDrehzahl-Kennlinie.

\title{
E. Ausblick
}

Die Untersuchungen dieser Arbeit haben gezeigt, daß man das zunächst recht kompliziert erscheinende Gebilde des wirklichen Motors mit ausgeprägten Polen und geschlossenen Streustegen in ein recht einfach zu übersehendes Modell zurückführen kann. Dabei sind nur unbedeutende Vernachlässigungen notwendig, die sich aber nachträglich durch Korrekturen erfassen lassen. Diese Arbeit behandelt zweisträngige Motoren bei Betrieb am symmetrischen Zweiphasennetz. Die gewonnenen Ergebnisse lassen leicht eine Erweiterung der Theorie auf dreisträngige Motoren zu.

In der Praxis werden solche Motoren meist als Kondensatormotoren am Einphasennetz betrieben. Dann sind zusätzliche theoretische Schwierigkeiten zu erwarten, wobei aber die Aussagen dieser Arbeit notwendige Grundlagen bieten. Gleichzeitig sollte es möglich sein, die hier gewonnenen Ergebnisse bei der Berechnung von Spaltpolmotoren mit geschlossenen Streustegen zu verwerten.

\section{Literatur}

[1] Hillebrand, F.: Zur Geschichte des Drehstromes. ETZ A 80 (1959) H. 13.

[2] Kocourek, K.: Streuleitfähigkeit geschlossener Läufernuten. E. u. M. 53 (1935) H. 36 .

[3]. Rothert, H.: Über die Nutstreuung elektrischer Maschinen. Arch. Elektrotechnik 32 (1938).

[4] TAEGen, F.: Über die Oberfeldmomente von Drehstromasynchronmotoren. Diss. TH Hannover 1961.

Dr.-Ing. INGO Koch, AEG Fabrik Oldenburg, 23 Oldenburg, Klingenbergplatz.

\section{Berichtigung}

zum Beitrag ,J. Frscher, Definitionen der Größen des magnetischen Feldes und die „Zurückführung auf absolutes Maß" nach C. F. Gauß" in Heft 1 des 49. Bandes, Seite 13:

In den ersten beiden Zeilen des ,Summary" muß es statt mantities richtig heißen: quantities. 